\title{
Geometrical Formalism for Dynamically Corrected Gates in Multiqubit Systems
}

\author{
Donovan Buterakos, ${ }^{1, *}$ Sankar Das Sarma,${ }^{1}$ and Edwin Barnes ${ }^{2}$ \\ ${ }^{1}$ Condensed Matter Theory Center and Joint Quantum Institute, Department of Physics, University of Maryland, \\ College Park, Maryland 20742-4111, USA \\ ${ }^{2}$ Department of Physics, Virginia Tech, Blacksburg, Virginia 24061, USA
}

(Received 17 September 2020; revised 22 January 2021; accepted 5 February 2021; published 9 March 2021)

\begin{abstract}
The ability to perform gates in multiqubit systems that are robust to noise is of crucial importance for the advancement of quantum information technologies. However, finding control pulses that cancel noise while performing a gate is made difficult by the intractability of the time-dependent Schrödinger equation, especially in multiqubit systems. Here, we show that this issue can be sidestepped by using a formalism in which the cumulative error during a gate is represented geometrically as a curve in a multidimensional Euclidean space. Cancelation of noise errors to leading order corresponds to closure of the curve, a condition that can be satisfied without solving the Schrödinger equation. We develop and uncover general properties of this geometric formalism, and derive a recursion relation that maps control fields to curvatures for Hamiltonians of arbitrary dimension. We demonstrate the utility of the formalism by employing it to design pulses that simultaneously correct against both noise errors and crosstalk for two qubits coupled by an Ising interaction. We give examples both of a single-qubit rotation and a two-qubit maximally entangling gate. The results obtained in this example are relevant to both superconducting transmon qubits and semiconductor quantum-dot spin qubits. We propose this geometric formalism as a general technique for pulse-induced error suppression in quantum computing gate operations.
\end{abstract}

DOI: 10.1103/PRXQuantum.2.010341

\section{INTRODUCTION}

Dynamical gate correction is an important topic in the field of quantum information technology because logical error-correction schemes require that the individual gate error be below a given threshold value [1]. Any quantum error-correction protocol can only be implemented after individual gate operations are sufficiently error-free, often necessitating dynamical decoupling of environmental noise by external pulses. Dynamically corrected gates rely on using precise control of the underlying Hamiltonian to perform rotations such that the error introduced by a given noise source at one point in the pulse will exactly cancel the error introduced by the same noise source at other points in the pulse. This idea, adopted from nuclear magnetic resonance (NMR) where pulses are used to reduce spin dephasing such as with the Hahn spin echo effect, is now used regularly to extend qubit coherence times [2-16].

\section{*dbuterak@umd.edu}

Published by the American Physical Society under the terms of the Creative Commons Attribution 4.0 International license. Further distribution of this work must maintain attribution to the author(s) and the published article's title, journal citation, and DOI.
Hahn spin echo and closely related multipulse sequences such as the Carr-Purcell-Meiboom-Gill sequence are primarily used to preserve quantum states. More sophisticated pulse sequences have also been proposed to perform quantum gate operations that are robust to various noise sources [17-28]. Many of these pulses are derived by finding a series of rotations that combine to produce the desired gate while canceling error to first order or higher. Finding such a sequence often involves numerically solving large systems of nonlinear equations to determine the parameters that define each of the smaller rotations. Solutions to these equations are not unique, and finding one errorcorrecting pulse sequence only amounts to finding a local maximum in fidelity, and thus many of the resulting pulse sequences are far from optimal. Additionally, many techniques rely on using the delta function or square pulses, neither of which can be precisely implemented in physical systems, as waveform generators have bandwidth and amplitude limitations. Thus, general methods for finding smooth, optimal dynamical-decoupling pulses are highly desirable.

One particularly powerful method for generating singlequbit error-correcting pulses is to represent these pulses as curves parameterized by the cumulative error at any given point in time. Zeng et al. [29] first showed that, for a simple Hamiltonian, pulses that correct against first-order error can be represented as closed curves in a plane, and 
that the driving field is proportional to the curvature of the curve. Conditions were given for canceling error at higher orders; specifically, for second order, the total signed area enclosed by the curve must equal zero. This formalism was used to derive the fastest possible pulses implementing specific single-qubit gates given constraints on the magnitude of the driving field [30]. In Ref. [31], this formalism was extended to more general single-qubit Hamiltonians with multiple control fields. Here pulses were represented as curves in three dimensions, with the strengths of the driving fields being related to the curvature and torsion of the curve. This formalism effectively extends earlier techniques for reverse-engineering solutions to the Schrödinger equation to the realm of dynamically corrected gates $[32,33]$. A prior geometric approach presented in Ref. [34] is in fact equivalent to that of Ref. [31], as shown in the latter. However, the method of Ref. [34] requires solving nonlocal and nonlinear constraints to obtain the desired waveforms, and it does not provide a way to cancel higher-order noise errors. Despite these limitations, the method of Ref. [34] does have the benefit of enabling the cancelation of multiple noise sources $[35,36]$. Additional techniques for reverse-engineering solutions to the time-dependent Schrödinger equation for dynamical decoupling purposes have been proposed, but these tend to require a higher degree of controllability over the qubit [37].

In this paper, we extend the geometric formalism to systems of multiple qubits subject to quasistatic noise. We show that many of the features of the single-qubit formalism carry over to the multiqubit case. Pulses are represented as curves in a higher-dimensional space, and those that cancel first-order error correspond to closed curves. The duration of each pulse is given by the length of the corresponding curve, and the amplitudes of the driving fields are related to the curvature coefficients at each point along the curve. As a demonstration, we derive errorcorrecting pulses for a class of two-qubit Hamiltonians using curves in six dimensions. We focus on Hamiltonians that describe Ising-type interactions commonly arising in both superconducting transmon qubits and semiconductor spin qubits. We demonstrate that our approach can be used to generate pulses for both single-qubit gates and multiqubit entangling gates, and we show that both interqubit crosstalk and quasistatic noise can be simultaneously suppressed by these pulses. We test the performance of these pulses with numerical simulations and confirm that the leading-order infidelity in the presence of noise is canceled.

This paper is organized as follows. In Sec. II, we derive the geometric formalism for a general Hamiltonian. In Sec. III, we give an example of a specific two-qubit system, and we derive pulses that implement single-qubit and two-qubit gates while suppressing noise and crosstalk. We conclude in Sec. IV.

\section{MULTIQUBIT GEOMETRICAL FORMALISM}

We consider a generic Hamiltonian $H_{0}(t)$ that contains time-dependent driving fields. Suppose that there is some error term $\delta H$ that is small compared to the scale of $H_{0}(t)$. In many qubit platforms including superconducting qubits and spin qubits, the time required to perform gates is generally much shorter than the time scale over which $\delta H$ varies [38-42]. In this case, even dynamic noise can be well approximated as quasistatic, meaning that the noise fluctuation $\delta H$ is treated as constant during a single pulse, but it can vary from one pulse to the next [20]. This is the situation where dynamical decoupling is most effective, and we employ this quasistatic approximation throughout this work. The full Hamiltonian is then $H(t)=H_{0}(t)+\delta H$. Our goal is to determine driving fields in $H_{0}(t)$ that perform a desired gate while canceling the effects of noise to leading order in $\delta H$. We consider the evolution operator in the interaction picture. Let $U(t)=\mathcal{T} e^{-i \int_{0}^{t} H(\tau) d \tau}$ be the time-dependent evolution operator of the full noisy Hamiltonian $H$, and let $R(t)=\mathcal{T} e^{-i \int_{0}^{t} H_{0}(\tau) d \tau}$ be the ideal noiseless evolution operator, where $\mathcal{T}$ represents the timeordering operator. This means that the time derivative of $R$ is

$$
\frac{d R}{d t}=-i H_{0} R
$$

Then the evolution operator in the interaction picture $U_{I}(t)$ satisfies $U=R U_{I}$, and the time-dependent Schrödinger equation becomes

$$
i \frac{d U_{I}}{d t}=H_{I} U_{I}=\left(R^{\dagger} \delta H R\right) U_{I}
$$

The solution to the Schrödinger equation to first order in $\delta H$ is given by

$$
U_{I}(t)=\mathcal{T} e^{-i \int_{0}^{t} H_{I}(\tau) d \tau}=\mathbb{1}-i \int_{0}^{t} H_{I}(\tau) d \tau+O\left(\delta H^{2}\right) .
$$

Note that the integral of $H_{I}$ is a measure of the total accumulated error at any time $t$, as the gate $U$ actually performed in the presence of noise is equal to the desired gate $R$ only if $U_{I}=\mathbb{1}$.

In order to help derive noise-canceling gates, we want to represent this traceless part of $U_{I}(t)$ as a curve in some $d$-dimensional space. To this end, we define $\mathcal{S}$ to be the vector space on which $H$ acts. For example, if $H$ describes an $n$-qubit system then $\mathcal{S}$ is the Hilbert space of $n$ qubits. The set of all traceless Hermitian matrices that act on $\mathcal{S}$ themselves form another vector space over $\mathbb{R}$, which we call $\mathcal{V}$ (e.g., $H$ will be an element of $\mathcal{V}$ ). In the case of $n$ qubits, $\mathcal{V}$ is the space of traceless $n$-qubit Pauli strings, 
which has dimension $\operatorname{dim} \mathcal{V}=4^{n}-1$. Define an inner product on this vector space as

$$
\vec{V} \cdot \vec{W}=\frac{1}{\operatorname{dim} \mathcal{S}} \operatorname{Tr} V W,
$$

where $\operatorname{dim} \mathcal{S}$ is the dimension of the vector space $\mathcal{S}$, and therefore equal to the size of the matrices in $\mathcal{V}$ (e.g., for the case of a system of $n$ qubits, $\operatorname{dim} \mathcal{S}=2^{n}$ ). Here, although $\vec{V}=V$, we use the notation $\vec{V}$, because in what follows, it helps to imagine that we expand $V$ in terms of a basis of Hermitian matrices (e.g., $n$-qubit Pauli strings) with real coefficients. If these coefficients are time dependent then they trace out a curve in a higher-dimensional Euclidean space. In what follows, this basis expansion will be kept implicit for the sake of generality and ease of notation. The inner product in Eq. (4) is invariant under unitary frame transformations, which will become important later.

The possible error terms that can be generated by any specific pulse from a Hamiltonian $H$ will span some subspace of $\mathcal{V}$, which we call $\mathcal{V}_{\text {err }}$. The subspace $\mathcal{V}_{\text {err }}$ is not universal and may or may not be equal to $\mathcal{V}$ depending on the degrees of freedom present in $H_{0}$ and on what rotations are possible. For example, for a single-qubit system, $\mathcal{V}$ is the three-dimensional space consisting of the $X, Y$, and $Z$ Pauli matrices. In Ref. [29], it was shown that, for a Hamiltonian with a single Pauli matrix, such as $H_{0}(t)=\Omega(t) X$, the error space $\mathcal{V}_{\text {err }}$ is a two-dimensional subspace of $\mathcal{V}$. In comparison, Ref. [31] examined a Hamiltonian with two different driving terms such as $H_{0}(t)=\Omega_{X}(t) X+\Omega_{Y}(t) Y$, and found that $\mathcal{V}_{\text {err }}$ in fact spans the entire space of $\mathcal{V}$ in this case. In general, $\mathcal{V}_{\text {err }}$ is the space spanned by $\delta H$ and all vectors to which $\delta H$ can be rotated by the evolution operator $R$ corresponding to a particular choice of $H_{0}(t)$. For example, consider a Hamiltonian of the form

$$
H_{0}(t)=\Omega_{1}(t) V_{1}+\Omega_{2}(t) V_{2}+\cdots+\Omega_{k}(t) V_{k},
$$

where the $V_{i}$ are products of Pauli matrices and $k$ is an arbitrary integer. Then $\mathcal{V}_{\text {err }}$ will include linear combinations of $\delta H$ and all vectors of the form $i\left[V_{i}, \delta H\right]$. Additionally, if any terms in $H_{0}$ do not commute with each other then the time ordering of $R$ will allow sequences of rotations, and thus nested commutators such as $-\left[V_{j},\left[V_{i}, \delta H\right]\right]$ will also be included in $\mathcal{V}_{\text {err }}$.

Let $d$ be the dimension of $\mathcal{V}_{\text {err }}$, and define a $d$ dimensional vector $\vec{G}(t)$ as

$$
\vec{G}(t)=\frac{1}{|\delta H|} \int_{0}^{t} H_{I}(\tau) d \tau .
$$

This gives the accumulated error present in Eq. (3) as a function of time, divided by $|\delta H|$ so that $\vec{G}(t)$ is independent of the actual strength of the noise. Here, the magnitude is given by $|\vec{A}|=\sqrt{\vec{A} \cdot \vec{A}}$ for any matrix $\vec{A}$. We can picture
$\vec{G}(t)$ as tracing out a curve in $\mathcal{V}_{\text {err }}$, with $\vec{G}(0)=0$. If $\vec{G}(t)$ returns to zero at some later time $t_{\text {pulse }}$ then the rotation performed will be unaffected by error to first order in $|\delta H|$, and thus dynamically corrected gates correspond to closed $d$-dimensional curves by construction. Now consider the time derivative of $\vec{G}(t)$ :

$$
\left|\frac{d \vec{G}}{d t}\right|=\frac{\left|H_{I}\right|}{|\delta H|}=1
$$

Since $d \vec{G} / d t$ always has unit length, the distance along the $d$-dimensional curve parameterized by $\vec{G}(t)$ corresponds to the time $t$ at that point. Thus, the tangent vector to the curve at any point is given by $d \vec{G} / d t$, and the normal vector is proportional to the second time derivative of $\vec{G}$. Using Eq. (1), we can express this in terms of $R, H_{0}$, and $\delta H$ as

$$
\begin{aligned}
\frac{d^{2} \vec{G}}{d t^{2}} & =\frac{1}{|\delta H|} \frac{d H_{I}}{d t} \\
& =\frac{1}{|\delta H|} \frac{d}{d t}\left(R^{\dagger} \delta H R\right) \\
& =\frac{i}{|\delta H|} R^{\dagger}\left[H_{0}, \delta H\right] R .
\end{aligned}
$$

The curvature $\kappa_{1}$ at any point along the curve can be calculated from this, and is given by

$$
\kappa_{1}=\left|\frac{d^{2} \vec{G}}{d t^{2}}\right|=\left|\frac{i}{|\delta H|} R^{\dagger}\left[H_{0}, \delta H\right] R\right|=\left|\frac{i}{|\delta H|}\left[H_{0}, \delta H\right]\right| .
$$

In this representation of the cumulative error $G(t)$ as a $d$ dimensional curve, the ideal evolution operator $R(t)$ is a rotation that takes the initial tangent vector $\delta H$ to the tangent vector $d \vec{G} / d t$ at any time $t$ along the curve. Because $R$ is a time-ordered exponential, it can be difficult to work with, since it cannot be analytically calculated for most choices of $H_{0}(t)$. However, as Eq. (9) shows, this does not prevent us from calculating the curvature, since the curvature at any point on a curve does not depend on the absolute orientation of the curve, but only on points in its own local neighborhood. This is reflected in Eq. (9) by the fact that $\kappa_{1}$ does not depend directly on $R$. Thus, for a given $\delta H$, the curvature provides a direct relationship between $G(t)$ and the Hamiltonian $H_{0}$, without the need to calculate $R$. If $d>2$, the same reasoning demonstrates that the higherorder curvature coefficients are independent of $R$ as well. These can be calculated using the Frenet-Serret equations

$$
\vec{e}_{n}=\frac{d^{n} \vec{G}}{d t^{n}}-\sum_{j=1}^{n-1}\left(\frac{d^{n} \vec{G}}{d t^{n}} \cdot \hat{e}_{j}\right) \hat{e}_{j} .
$$

Here, the vectors $\vec{e}_{n}$ (more precisely their normalized counterparts $\hat{e}_{n}$ ) define the Frenet-Serret frame at each point 
along the curve defined by $\vec{G}$. The number of vectors is therefore equal to $d$, the dimension of $\mathcal{V}_{\text {err }}$. In the singlequbit case for example where $d=3, \vec{e}_{1}, \vec{e}_{2}$, and $\vec{e}_{3}$ are the tangent, normal, and binormal vectors, respectively. Knowing how these vectors evolve in time is equivalent to knowing how the curve moves through space. These vectors evolve in an interdependent way that is governed by the generalized curvatures

$$
\kappa_{n}=\frac{d \hat{e}_{n}}{d t} \cdot \hat{e}_{n+1}
$$

In the single-qubit case, $\kappa_{1}$ is the curvature of the threedimensional space curve, while $\kappa_{2}$ is its torsion. More generally, $d-1$ curvatures are needed to characterize a curve in $d$ dimensions. The higher-order derivatives of $\vec{G}(t)$ can be calculated in a similar fashion to Eq. (8) above, which will generate nested commutators. There will, however, be additional terms due to the time dependence of $H_{0}$. Define the operator $C$ which acts on a matrix $V$ as

$$
C V=i\left[H_{0}, V\right] \text {. }
$$

Then the derivatives of $\vec{G}$ are given by

$$
\frac{d^{n} \vec{G}}{d t^{n}}=\frac{1}{|\delta H|} R^{\dagger}\left[\left(C+\frac{\partial}{\partial t}\right)^{n-1} \delta H\right] R .
$$

It is important to note that $C$ does not commute with $\partial / \partial t$, and thus there will be increasingly more terms in this expression for higher values of $n$. Equations (10)-(13) relate the generalized curvatures to the driving fields.

While Eq. (13) may not be easy to work with in general, there are specific conditions under which it can be simplified. Specifically, suppose that the following anticommutation relation holds: $\left\{H_{0}, \delta H\right\}=0$. Note that if $\delta H$ is proportional to a Pauli string then one can always transform to a basis in which this relation holds [31]. For convenience, define $Q=\delta H /|\delta H|$, and assume that $Q^{2}=1$. Define the operator $A_{n}$ such that the Frenet-Serret basis vectors can be written as

$$
\hat{e}_{n}=R^{\dagger} A_{n} Q R \text {. }
$$

Differentiating Eq. (14) yields

$$
\dot{\hat{e}}_{n}=i R^{\dagger}\left\{H_{0}, A_{n}\right\} Q R+R^{\dagger} \dot{A}_{n} Q R .
$$

On the other hand, the Frenet-Serret equations read

$$
\dot{\hat{e}}_{n}=\kappa_{n} \hat{e}_{n+1}-\kappa_{n-1} \hat{e}_{n-1} \text {. }
$$

We now show that the two terms in Eq. (15) directly correspond to the two terms in the Frenet-Serret equations.
To do this, we show that $\left[A_{n}, Q\right]=0$ for $n \bmod 4=0$ or 1 , and $\left\{A_{n}, Q\right\}=0$ for $n \bmod 4=2$ or 3 . We make use of the property that, for any operators $O_{1}$ and $O_{2}$ such that $O_{1}$ commutes with $Q$ and $O_{2}$ anticommutes with $Q$, the following condition holds:

$$
\operatorname{Tr} O_{1} Q O_{2} Q=0 .
$$

Consider the first Frenet-Serret vector (the tangent vector to the curve)

$$
\hat{e}_{1}=R^{\dagger} Q R
$$

Here $A_{1}=\mathbb{1}$, and thus $\left[A_{1}, Q\right]=0$ holds trivially. Differentiating $\hat{e}_{1}$, we obtain the second vector

$$
\dot{\hat{e}}_{1}=\kappa_{1} \hat{e}_{2}=i R^{\dagger}\left\{H_{0}, A_{1}\right\} Q R,
$$

from which we find that $\kappa_{1} A_{2}=i\left\{H_{0}, A_{1}\right\}$, and the relationship $\left\{A_{2}, Q\right\}=0$ follows. Similarly, we can consider the derivative of the $n$th vector given by Eq. (15). If $A_{n}$ commutes with $Q$ then $\dot{A}_{n}$ will also commute with $Q$, and $\left\{H_{0}, A_{n}\right\}$ will anticommute with $Q$. Similarly, if $A_{n}$ anticommutes with $Q$ then $\dot{A}_{n}$ will anticommute with $Q$, and $\left\{H_{0}, A_{n}\right\}$ will commute with $Q$. In either case, Eq. (17) implies that the two terms in Eq. (15) will be orthogonal to one another. Additionally, one of these two terms will be orthogonal to $\hat{e}_{n-1}$. This means that terms in Eq. (15) can be matched to the terms in Eq. (16), and the curvatures and operators $A_{n}$ can be obtained. Specifically, the following recursion relation holds:

$$
\kappa_{n} A_{n+1}= \begin{cases}i\left\{H_{0}, A_{n}\right\} & \text { if } n \text { is odd } \\ \dot{A}_{n} & \text { if } n \text { is even. }\end{cases}
$$

The curvatures are obtained by taking the magnitude of this expression, since $A_{n}$ has unit magnitude. Equation (20) thus provides a general mapping between control fields in the Hamiltonian and curvature coefficients of the curve.

\section{EXAMPLE: PULSES THAT CORRECT AGAINST NOISE AND CROSSTALK}

In this section, we apply the general geometrical formalism derived in Sec. II to a specific two-qubit Hamiltonian, and use it to derive pulses that dynamically decouple the two qubits from each other while suppressing quasistatic noise.

As an example, consider the case of two superconducting transmon qubits coupled either directly through a capacitor or indirectly via a microwave transmission line resonator [43-46]. In the weak-coupling regime and when the qubits are far detuned from one another, the system is 
described by the effective Hamiltonian [47-51]

$$
\begin{aligned}
H_{\mathrm{eff}}= & \frac{1}{2}\left(-\omega_{1}+\eta-\frac{1}{2} \lambda_{Z Z}\right) Z_{1} \\
& +\frac{1}{2}\left(-\omega_{2}-\eta-\frac{1}{2} \lambda_{Z Z}\right) Z_{2}+\frac{1}{4} \lambda_{Z Z} Z_{1} Z_{2} .
\end{aligned}
$$

Here, the $Z_{i}$ are Pauli matrices, $\omega_{i}$ is the energy splitting of the $i$ th transmon, $\eta$ is a coupling-induced frequency shift, and $\lambda_{Z Z}$ is a residual Ising-type interaction. The qubit frequencies are typically of the order of $1-10 \mathrm{GHz}$, while $\eta$ and $\lambda_{Z Z}$ are in the range $1-10 \mathrm{MHz}$. In the case of a direct, capacitive coupling, the latter two quantities are given by

$$
\begin{gathered}
\eta=\frac{g^{2}}{\omega_{2}-\omega_{1}}, \\
\lambda_{Z Z}=\frac{g^{2}}{\omega_{2}-\omega_{1}+\alpha_{1}}+\frac{g^{2}}{\omega_{1}-\omega_{2}+\alpha_{2}},
\end{gathered}
$$

where $g$ is the bare coupling and $\alpha_{i}$ is the anharmonicity of transmon $i$. Analogous expressions hold in the case of resonator-mediated coupling [43]. The Hamiltonian $H_{0}$ describes how the system evolves in the "idling" regime, where ideally the qubits would not interact with one another. An outstanding issue in the idling regime is the presence of the residual $Z Z$ coupling, $\lambda_{Z Z}$, which can produce unwanted entanglement and lead to coherent errors. This is a form of interqubit crosstalk, which is a significant issue in multiqubit processors $[47,48,50,52,53]$. We note that the $Z Z$ coupling can also be used to implement entangling gates by strongly driving one qubit (see, e.g., Refs. [25,26,44,49,51-54]). Alternatively, two-qubit gates can be implemented by tuning one of the qubits into resonance with the other to temporarily enhance the interaction. Here, we first show how to use the generalized geometric formalism to design pulses that dynamically decouple a qubit from a neighboring, noisy qubit. We then show a robust maximally entangling gate produced using the same method.

For concreteness, suppose that qubit 1 is a fixedfrequency transmon, while qubit 2 is a tunable transmon. The latter is generally more sensitive to magnetic flux noise [46,55-57]. Our goal is to decouple qubit 1 from qubit 2 despite the always-on $Z Z$ interaction. We do this by applying a pulse on qubit 2 that effectively unwinds the effects of both the residual $Z Z$ coupling and the quasistatic noise errors afflicting qubit 2 . The driving term is given by

$$
H_{\text {drive }}=\Omega(t) \cos \left(\omega_{d} t\right) X_{2},
$$

where $\Omega(t)$ is the pulse envelope and $\omega_{d}$ is the driving frequency. Applying the rotating-wave approximation and transforming to the frame of the drive using
$T=\operatorname{diag}\left(e^{-i \omega_{d} t}, 1,1, e^{i \omega_{d} t}\right)$, the total noiseless Hamiltonian becomes

$$
H_{\text {total }}=T\left(H_{0}+H_{\text {drive }}\right) T^{\dagger}-i T \dot{T}^{\dagger}=H_{1}+H_{12},
$$

where $H_{1}=\frac{1}{2}\left(\omega_{d}-\omega_{1}+\eta-\frac{1}{2} \lambda_{Z Z}\right) Z_{1}$ and

$$
\begin{aligned}
H_{12}= & \Omega(t) X_{2}+\frac{E_{1}+E_{2}}{2} Z_{2}+\frac{E_{1}-E_{2}}{2} Z_{1} Z_{2} \\
= & \left(\begin{array}{cccc}
E_{1} & \Omega(t) & 0 & 0 \\
\Omega(t) & -E_{1} & 0 & 0 \\
0 & 0 & E_{2} & \Omega(t) \\
0 & 0 & \Omega(t) & -E_{2}
\end{array}\right),
\end{aligned}
$$

with $E_{1}=\frac{1}{2}\left(\omega_{d}-\omega_{2}-\eta\right)$ and $E_{2}=\frac{1}{2}\left(\omega_{d}-\omega_{2}-\eta-\right.$ $\left.\lambda_{Z Z}\right)$. The noise Hamiltonian is given by $\delta H=\epsilon Z_{2}$, where $\epsilon$ is a stochastic noise parameter describing a fluctuation in the frequency of qubit 2 due to magnetic flux noise. We assume that $\epsilon$ remains constant during the pulse. This quasistatic noise model works well because flux noise is concentrated at low frequencies [46,55-57]. We note in passing that $H_{\text {total }}$ can also be used to describe a pair of semiconductor spin qubits, both for exchange-based [36,58-63] and capacitive [64-67] interqubit couplings. Similar issues with residual $Z Z$ couplings also arise in these systems, and the results we obtain below can be applied in that context as well. In this case, $\delta H$ can be interpreted as Zeeman frequency fluctuations caused by hyperfine interactions with nuclear spins [68].

Since $\left[H_{1}, H_{12}\right]=0$, the evolution operator factorizes, $U_{\text {total }}=e^{-i H_{1} t} U_{12}$, where $U_{12}$ is the evolution operator generated by $H_{12}$. We therefore focus solely on $U_{12}$ since it contains all the crosstalk and noise effects. The goal is to engineer the pulse so that $U_{12}\left(t_{\text {pulse }}\right)=e^{i \chi_{1} Z_{1}} \otimes e^{i \chi_{2} Z_{2}}$, where $\chi_{1}, \chi_{2}$ are independent of $\epsilon$. While our objective is to preserve the states of both qubits, we allow for the possibility that local $Z$ gates are implemented on each qubit since these are easily undone by classical postprocessing [46]. (Note that $e^{-i H_{1} t_{\text {pulse }}}$ and the transformation $T$ also implement local $Z$ gates.) The error curve associated with $U_{12}$ is given by $\vec{G}(t)=\int_{0}^{t} d \tau U_{12}^{\dagger}(\tau) Z_{2} U_{12}(\tau)$. If we consider all possible commutators of the terms in $H_{12}$ and $\delta H$, we find that the error curve lives in the six-dimensional space $\mathcal{V}_{\text {err }}$ spanned by $\left\{X_{2}, Y_{2}, Z_{2}, Z_{1} X_{2}, Z_{1} Y_{2}, Z_{1} Z_{2}\right\}$. Using Eq. (20), we calculate the five curvature coefficients as

$$
\begin{gathered}
\kappa_{1}=2|\Omega|, \\
\kappa_{2}=\sqrt{2\left(E_{1}^{2}+E_{2}^{2}\right)}, \\
\kappa_{3}=\frac{\sqrt{2}\left|E_{1}^{2}-E_{2}^{2}\right|}{\sqrt{E_{1}^{2}+E_{2}^{2}}},
\end{gathered}
$$




$$
\begin{gathered}
\kappa_{4}=2 \sqrt{\Omega^{2}+\frac{2 E_{1}^{2} E_{2}^{2}}{E_{1}^{2}+E_{2}^{2}}}, \\
\kappa_{5}=\frac{E_{1} E_{2} \sqrt{2\left(E_{1}^{2}+E_{2}^{2}\right)}}{\Omega^{2}\left(E_{1}^{2}+E_{2}^{2}\right)+2 E_{1}^{2} E_{2}^{2}} \frac{d \Omega}{d t} .
\end{gathered}
$$

Every pulse $\Omega(t)$ corresponds to some six-dimensional curve given by these curvature coefficients. It is important to realize that the converse is not true: every sixdimensional curve does not necessarily correspond to a pulse that can be created by the Hamiltonian given by Eq. (26). This is because there are in general five degrees of freedom corresponding to a six-dimensional curve (corresponding to the five curvature coefficients), but in Eq. (26) we have constrained the form of the Hamiltonian such that it has only one independent driving field. Because the Hamiltonian given by Eq. (26) is block diagonal, the system can alternatively be treated as two independent $2 \times 2$ subsystems corresponding to the two blocks, with the constraint that the driving field $\Omega$ must be the same in each block. These subsystems can each be treated using the single-qubit formalism given in Ref. [31]. This means that a closed six-dimensional curve that satisfies Eqs. (26) can be mapped to two separate three-dimensional closed curves with equal lengths and curvatures, but different torsions given by $-2 E_{1}$ and $-2 E_{2}$.

Before we design a pair of curves with equal curvatures and differing torsions, it is worth considering whether we could instead just treat the $Z Z$ coupling at the same footing as quasistatic noise. This is possible when $\left|E_{1}-E_{2}\right| \ll$ $t_{\text {pulse }}^{-1}$, where $t_{\text {pulse }}$ is the duration of the pulse used to implement the decoupling. Since $\kappa_{3}$ is proportional to $\left|E_{1}-E_{2}\right|$, it will be small, and thus the entire curve will lie close to a three-dimensional subspace of the six-dimensional space. We could then use the single-qubit formalism of Ref. [31] inside this subspace to obtain closed error curves to leading order in $\left|E_{1}-E_{2}\right| t_{\text {pulse }}$. This works because the single-qubit formalism is designed to correct against small $Z$ errors, so if $E_{1}$ is close to $E_{2}$ then $H_{0}$ can be treated as two copies of the same $2 \times 2$ Hamiltonian, and the $Z_{1} Z_{2}$ term can be included with the error in each case. To determine whether or not this is a viable option, we need to consider typical values of $\left|E_{1}-E_{2}\right|=\left|\lambda_{z Z}\right| / 2$. For $\left|\lambda_{z Z}\right|=10$ $\mathrm{MHz}$, we would need $t_{\text {pulse }} \ll 30 \mathrm{~ns}$. Considering that gate times are typically of the order of tens to hundreds of nanoseconds in transmon systems $[52,69,70]$, this condition is not easily met. Thus, we do not assume that $\left|E_{1}-E_{2}\right| t_{\text {pulse }}$ is small, and we instead focus on the problem of designing two distinct but related three-dimensional error curves.

The task is then to find two three-dimensional curves with the same length and curvature, but with different constant torsions. There has been much work on developing methods of generating closed curves of constant torsion [71-74]; however, the analytic methods often used are not applicable here due to the additional constraint that the two curves must have equal curvatures. Thus, we instead approach the problem by numerically piecing together sets of helices to make two smooth closed curves. Since a helix has constant torsion, it is straightforward to impose the condition that the two curves have torsions fixed at $-2 E_{1}$ and $-2 E_{2}$. Helices also have constant curvature, which means that a closed curve comprised of connected helices corresponds to a series of square pulses that collectively cancel errors in a manner similar to the SUPCODE method for designing dynamically corrected gates [20]. Alternatively, in the six-dimensional picture, higher-dimensional generalizations of helices (curves with constant curvature coefficients) can be used. These will be five dimensional, since $\kappa_{5}$ is zero, due to $d \Omega / d t$ vanishing for a square pulse. Care must be taken for the step function transition between different values of $\Omega$, as $d \Omega / d t$ becomes a delta function at these points. At these transitions, $\Omega(t)$ can be treated as a steep constant slope over a short interval of time $\delta$, in the limit where $\delta \rightarrow 0$. In this case $\kappa_{1}$ through $\kappa_{4}$ will be finite, and thus will have no effect on the curve as $\delta \rightarrow 0 . \kappa_{5}$ will have a delta function contribution, resulting in a rotation between $\hat{e}_{5}$ and $\hat{e}_{6}$ at the point along the curve corresponding to the transition. The exact angle of rotation $\phi$ corresponding to a step from $\Omega_{1}$ to $\Omega_{2}$ is given by

$$
\phi=\int_{\Omega_{1}}^{\Omega_{2}} \frac{E_{1} E_{2} \sqrt{2\left(E_{1}^{2}+E_{2}^{2}\right)}}{\Omega^{2}\left(E_{1}^{2}+E_{2}^{2}\right)+2 E_{1}^{2} E_{2}^{2}} d \Omega .
$$

We choose to consider curves that are $n$-fold rotationally symmetric, as these curves are easier to visualize and require fewer numerical constraints to be fulfilled. To this end, we consider one curve segment where the Frenet-Serret frame at one endpoint is equal to the FrenetSerret frame at the other endpoint after having undergone a relative $2 k \pi / n$ rotation, for any integer $k$ coprime to $n$. Any curve segment of this form will produce a closed curve when repeated $n$ times, provided that the displacement vector between endpoints lies within the plane of rotation. The constraint that the displacement vector must lie within a given plane is easier to fulfil than the corresponding constraint for nonsymmetric closed curves, that the displacement vector must equal 0 exactly. Thus, it is advantageous to use symmetric curves when possible, as they reduce the total constraints needed to produce a closed curve. For our numerics, we consider threefold symmetric curves, which means that the resulting pulse will be a three-part composite pulse, with all three parts identical. To produce these curves, we numerically adjust the length and curvature of each helix until the conditions on the displacement vector and 

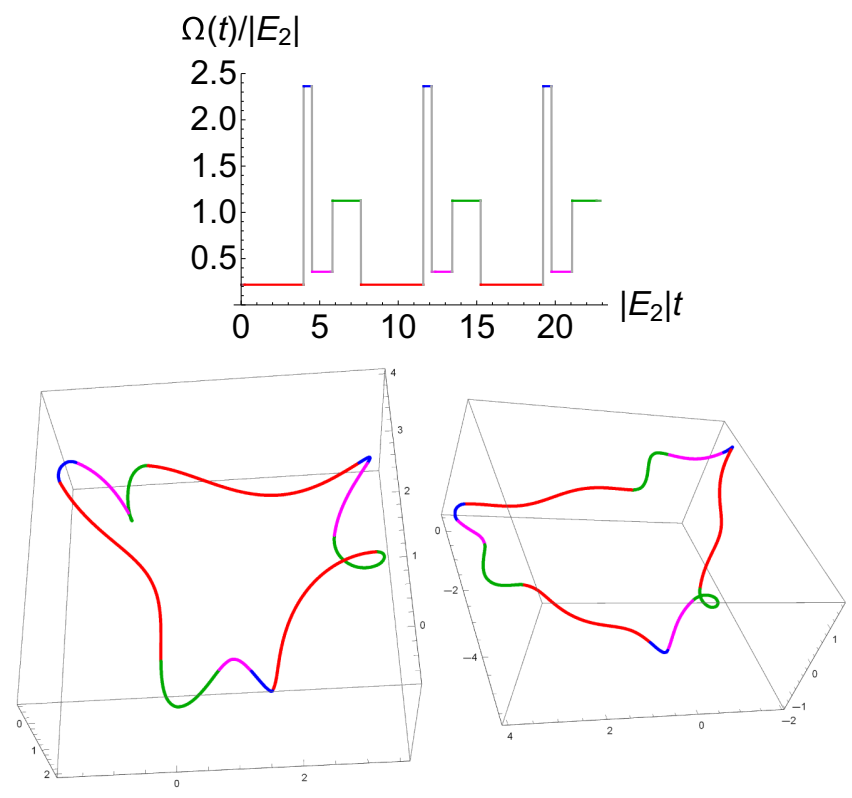

FIG. 1. Top: a square pulse sequence implementing a $Z_{1}$ gate that dynamically cancels error to first order in the noise strength $\epsilon$ for the Hamiltonian given by Eq. (26). Bottom: error curves for the two $2 \times 2$ blocks of the Hamiltonian. These are curves of constant torsion, with torsions $-2 E_{1}$ and $-2 E_{2}=-4 E_{1}$ for the left and right, respectively. They are comprised of helices, and are colored to demonstrate which part of the curve corresponds to which part of the pulse in the top part of the figure.

the Frenet-Serret frames at the endpoints are met. In Fig. 1 we show an example of a composite square pulse derived in this way for the case $E_{2}=2 E_{1}$. This relation between $E_{1}$ and $E_{2}$ corresponds to setting the drive frequency to

$$
\omega_{d}=\omega_{2}+\eta-\lambda_{Z Z}
$$

From Fig. 1, we see that the pulse duration is $t_{\text {pulse }} \approx$ $23 /\left|E_{2}\right|$, which is the time at which the crosstalk and noise effects are removed. Therefore, $t_{\text {pulse }}$ should be chosen to equal the desired idling time. For this particular example where $\left|E_{2}\right|=2\left|E_{1}\right|=\left|\lambda_{Z Z}\right|$, we have $t_{\text {pulse }} \approx$ $23 /\left|\lambda_{Z Z}\right|$. For $\left|\lambda_{z Z}\right|=10 \mathrm{MHz}$, this corresponds to $t_{\text {pulse }} \approx$ 360 ns. This timescale can be adjusted by changing the ratio $E_{2} / E_{1}$ and by changing $\lambda_{Z Z}$ through its dependence on the tunable frequency $\omega_{2}$ [see Eq. (23)]. The latter amounts to rescaling the curves and, hence, the pulse amplitude.

In experiments, square pulses cannot be exactly performed, since pulse generators have bandwidth and pulse rise-time limitations. However, we can numerically search for a smooth pulse similar in shape to the square pulse sequence already obtained. We do this by choosing a pulse shape of the form

$$
\begin{aligned}
\Omega(t)= & c_{0}+\frac{c_{1}}{1+a_{1}^{2} \sin ^{2}\left(\pi t / t_{p}+\phi_{1}\right)} \\
& +\frac{c_{2}}{1+a_{2}^{2} \sin ^{2}\left(\pi t / t_{p}+\phi_{2}\right)} .
\end{aligned}
$$

The form is meant to approximate a Lorentzian pulse, except that it is periodic with period $t_{p}$. The curves can then be numerically generated, and the parameters in Eq. (30) adjusted until closed curves are obtained. Because we use more parameters than the dimension of the space, solutions that produce closed curves are not necessarily unique. Uniqueness is not essential here since all we need is a solution providing dynamical decoupling, and the fact that there may be other solutions is not a problem. If finding solutions inside a desired parameter regime is difficult, a third Lorentzian can be added to the pulse to allow for more parameters. Adding more parameters should simplify the process of finding a possible solution as long as we do not insist on unique solutions; however, the shape of the resulting pulse will be more complicated as more parameters are used. Using this method, we find the pulse and curves shown in Fig. 2. The parameter values used to produce these results are given in Table I. The total pulse time is $t_{\text {pulse }}=3 t_{p} \approx 21.3 /\left|E_{2}\right|$. In Fig. 3, we plot
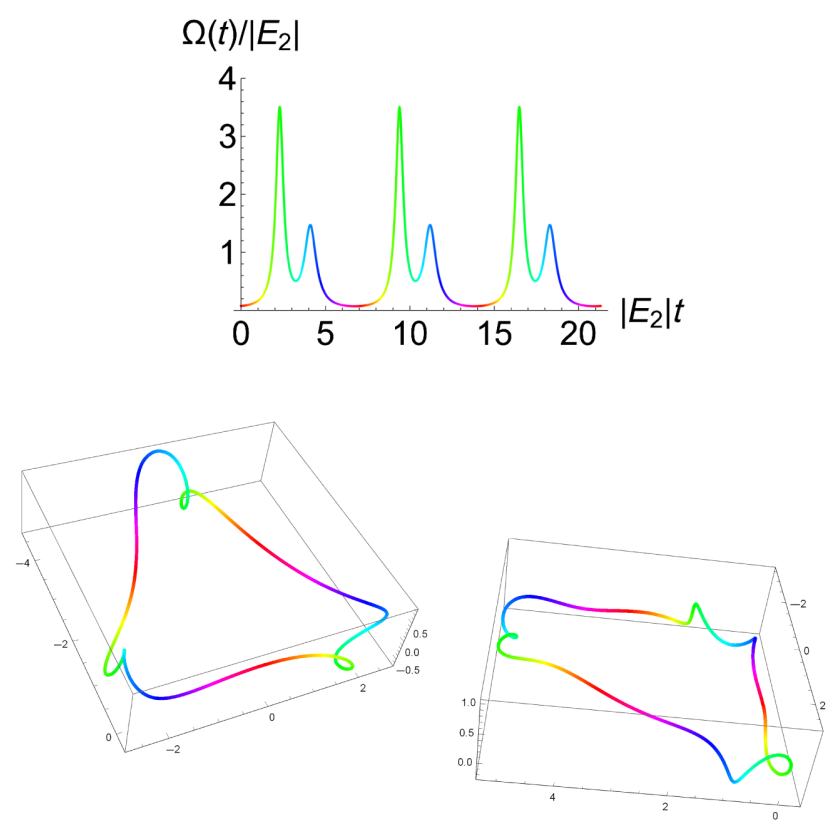

FIG. 2. Top: a smooth pulse implementing a $Z_{1}$ gate that dynamically cancels error to first order in the noise strength $\epsilon$ for the Hamiltonian given by Eq. (26). This pulse is of the form given by Eq. (30) with parameter values shown in Table I. Bottom: error curves for the two $2 \times 2$ blocks of the Hamiltonian. These are curves of constant torsion, with torsions $-2 E_{1}$ and $-2 E_{2}=-4 E_{1}$ for the left and right, respectively. The curves are colored to illustrate which points correspond to which part of the pulse in the top part of the figure. 
TABLE I. Parameter values used in Eq. (30) to produce the pulse in Fig. 2.

\begin{tabular}{lc}
\hline \hline$c_{0}$ & -0.0835558 \\
$c_{1}$ & 6.91751 \\
$c_{2}$ & 2.84417 \\
$a_{1}$ & 8.46347 \\
$a_{2}$ & 5.40744 \\
$\phi_{1}$ & -1.01143 \\
$\phi_{2}$ & -1.81521 \\
$t_{p}$ & 7.09776 \\
\hline \hline
\end{tabular}

the error curve in the six-dimensional representation of the same pulse by projecting it into two three-dimensional subspaces. We see that it retains its threefold rotational symmetry. In two of these dimensions, $\vec{e}_{1}(0)$ and $\vec{e}_{4}(0)$, the curve covers much more distance than in the other four.

By looking at each $2 \times 2$ block in Eq. (26) individually, we can use the same method as for the single-qubit case to determine the gates performed. For either $2 \times 2$ block, the evolution operator can be parameterized as [31]

$$
U_{12}^{\text {block }}=\left(\begin{array}{cc}
e^{i \varphi / 2} \cos (\chi / 2) & -i e^{-i \varphi / 2} \sin (\chi / 2) \\
-i e^{i \varphi / 2} \sin (\chi / 2) & e^{-i \varphi / 2} \cos (\chi / 2)
\end{array}\right)
$$

and the tangent vector of the corresponding threedimensional curve is

$$
\hat{e}_{1}=(-\sin \chi \sin \varphi, \sin \chi \cos \varphi, \cos \chi) .
$$

Setting $\chi(0)=0=\varphi(0)$ ensures that $U_{12}^{\text {block }}(0)=\mathbb{1}$. For symmetric closed curves like those shown in Figs. 2 and 3, the Frenet-Serret frame must match at the initial and final times: $\hat{e}_{1}\left(t_{\text {pulse }}\right)=\hat{e}_{1}(0)$, and similarly for $\hat{e}_{2}$ and $\hat{e}_{3}$, where these vectors belong to the three-dimensional spaces corresponding to each block. This then requires $\chi\left(t_{\text {pulse }}\right)=$ $2 \pi m$ and $\varphi\left(t_{\text {pulse }}\right)=2 \pi n$, where $m$ and $n$ are integers.

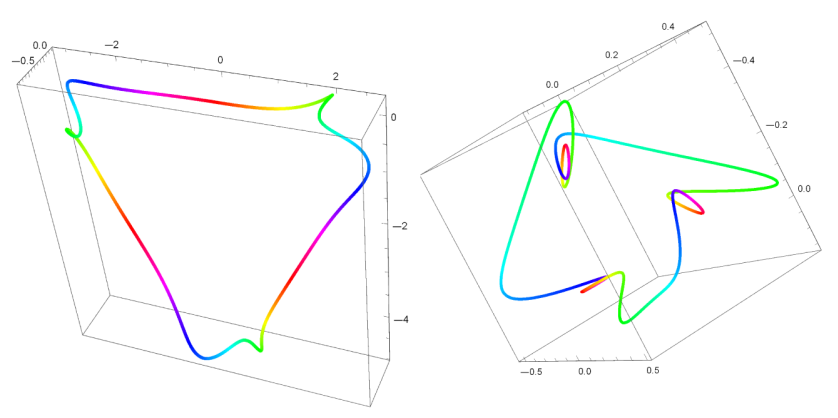

FIG. 3. Left: projection of the six-dimensional error curve for the pulse in Fig. 2 onto the $\vec{e}_{1}(0), \vec{e}_{3}(0), \vec{e}_{4}(0)$ subspace. The two largest dimensions correspond to $\vec{e}_{1}(0)$ and $\vec{e}_{4}(0)$. Right: projection of the six-dimensional curve onto the $\vec{e}_{2}(0), \vec{e}_{5}(0), \vec{e}_{6}(0)$ subspace. Note that the length of the curve in these dimensions is much smaller than in dimensions 1 and 4 on the left, and is comparable to dimension 3 on the left.
Thus, pulses that lead to symmetric closed curves can only perform identity operations in each of the $2 \times 2$ blocks: $U_{12}^{\text {block }}\left(t_{\text {pulse }}\right)=(-1)^{m+n} \mathbb{1}$. These identity operations can have a different relative sign, resulting in a $Z_{1}$ gate, as is the case with the pulse shown in Fig. 2.

Removing the symmetry requirement will generate other gates, including both single-qubit and two-qubit entangling gates. For example, an entangling controlled$Z$ (CZ) gate can be generated from a curve for which the Frenet-Serret frame at $t=t_{\text {pulse }}$ is twisted by angle $\pi$ about $\hat{e}_{1}(0)$ so that $\hat{e}_{1}\left(t_{\text {pulse }}\right)=\hat{e}_{1}(0), \hat{e}_{2}\left(t_{\text {pulse }}\right)=-\hat{e}_{2}(0)$, $\hat{e}_{3}\left(t_{\text {pulse }}\right)=-\hat{e}_{3}(0)$. Nondiagonal gates such as the controlled NOT (CNOT) gate require at least one of the curves to have a cusp at the initial or final point, because, for these gates, $\chi\left(t_{\text {pulse }}\right) \neq 2 \pi m$, and so $\hat{e}_{1}\left(t_{\text {pulse }}\right)$ will not be parallel to $\hat{e}_{1}(0)$. It is important to note that a periodic pulse will not necessarily lead to a symmetric curve, and in fact will only do so if the Frenet-Serret frame undergoes the proper rotation after one period of the pulse. This means that in order to produce a CZ or CNOT gate, one can attempt to find a pulse that in one $2 \times 2$ block produces a corrected $X$ or $Z$ gate, and in the other $2 \times 2$ block satisfies the rotation requirements on the Frenet-Serret frame. Then repeating this pulse 3 times will cause the first block to undergo a $X^{3}=X$ or $Z^{3}=Z$ rotation, and will cause the second block to form a closed symmetric curve, resulting
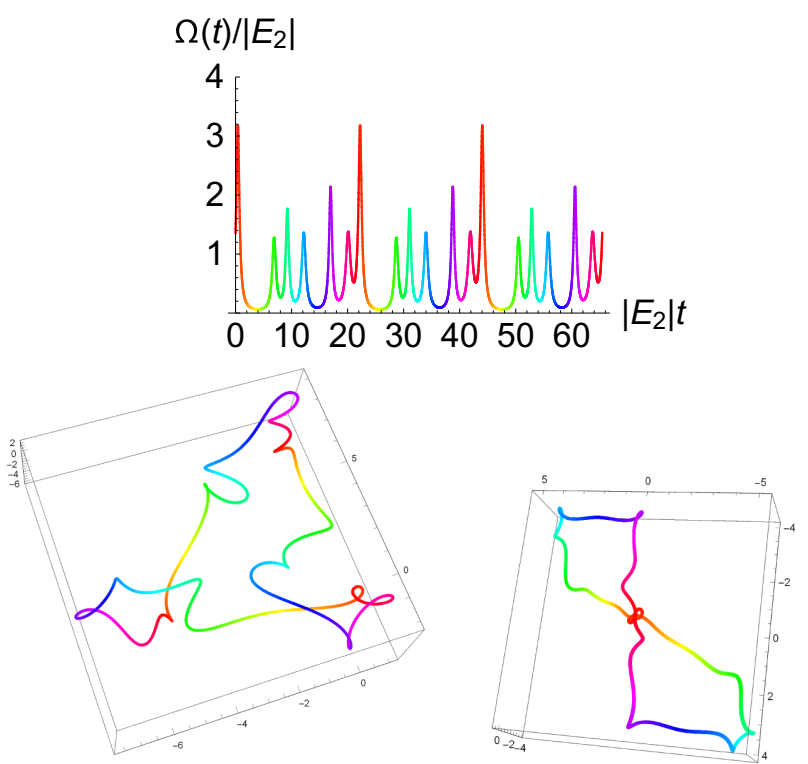

FIG. 4. Top: a smooth pulse implementing the CNOT-like gate given by Eq. (33), which dynamically cancels error to first order in the noise strength $\epsilon$ for the Hamiltonian given by Eq. (26). This pulse is of the form similar to Eq. (30) but extended to include more Lorentzians. Bottom: error curves for the two $2 \times 2$ blocks of the Hamiltonian. These are curves of constant torsion, with torsions $-2 E_{1}$ and $-2 E_{2}=-4 E_{1}$ for the left and right, respectively. The curves are colored to illustrate which points correspond to which part of the pulse in the top part of the figure. 
in an identity operation. Thus, the total two-qubit operation will be a CNOT or $\mathrm{CZ}$ gate, respectively, up to a phase difference between the two blocks. In Fig. 4 we show an error-correcting pulse produced by this method that implements the gate

$$
R=\left(\begin{array}{cccc}
0 & -i & 0 & 0 \\
-i & 0 & 0 & 0 \\
0 & 0 & 1 & 0 \\
0 & 0 & 0 & 1
\end{array}\right)
$$

This gate is equivalent to a CNOT gate up to a single-qubit $Z_{1}$ rotation.

In order to demonstrate that these pulses do properly correct against error, we numerically calculate the infidelity of the gate using the full noisy Hamiltonian for noise strengths $\epsilon$ ranging across several orders of magnitude. We plot the resulting infidelity versus the noise strength in Fig. 5. We define infidelity as $|U-R|$, where $U$ is the noisy gate and $R$ is the ideal gate (in this case $Z_{1}$ ). We see that the infidelity scales as the square of the noise strength, indicating that the gates cancel error to first order in $\epsilon$. In the case where $\left|E_{2}\right|=\left|\lambda_{z Z}\right|=10 \mathrm{MHz}$, the infidelity is reduced by $2-3$ orders of magnitude for noise strengths in the range 10-100 kHz. Thus, the geometrically designed
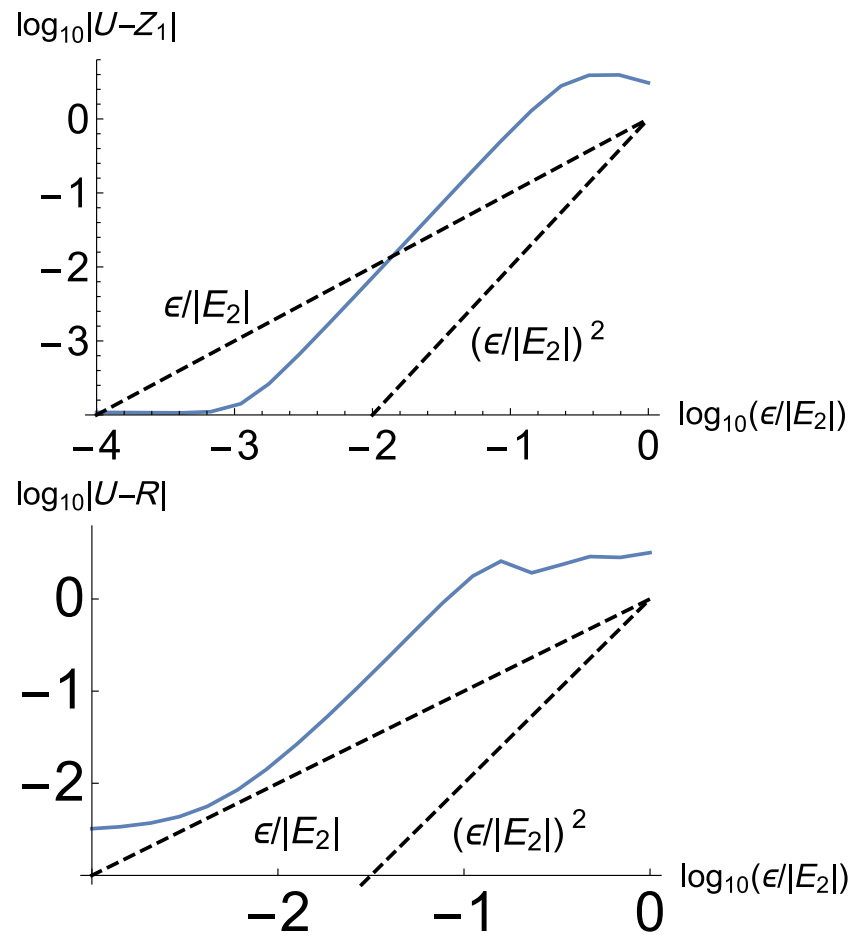

FIG. 5. Infidelity versus noise strength $\epsilon$ (solid blue curve) for the pulses shown in Fig. 2 (top) and Fig. 4 (bottom). The dashed lines show linear and quadratic scaling with noise strength for comparison. It is clear that the gate infidelity is consistent with quadratic scaling, indicating that first-order noise errors have been canceled. pulse indeed corrects against noise while suppressing the unwanted $Z Z$ coupling, as expected.

\section{CONCLUSION}

We show how to generalize the geometric formalism for producing dynamically corrected single-qubit gates to multiqubit systems. Like the single-qubit case, the cumulative first-order error can be represented as a curve in Euclidean space. Distance along the curve corresponds to the elapsed time from the beginning of the pulse, and the amplitudes of the driving fields can be related to the curvature coefficients at each point on the curve. Critically, using these curvature coefficients circumvents the need to evaluate the time-ordered exponential of the Hamiltonian, which in general cannot be done analytically except in very special cases. We present equations that show how to calculate these curvature coefficients in terms of the time derivatives and commutators of the noiseless and error Hamiltonians. We use this formalism to derive pulses that simultaneously suppress crosstalk and dephasing noise in qubits coupled by always-on Ising interactions. Such residual interactions are a common nuisance in both superconducting qubits and semiconductor spin qubits. Specifically, we give examples of pulses that implement single-qubit gates and multiqubit entangling gates, and we demonstrate that these pulses cancel first-order noise errors by computing the infidelity of the gate as a function of the noise strength.

While this formalism provides a good starting point for deriving dynamically corrected gates for multiqubit systems, there are still several challenges. In particular, it is usually the case that only a few terms in the Hamiltonian can be controlled dynamically. In this case, we need to restrict to the subset of curves that produce Hamiltonians of the desired form. This issue arises when the number of control fields is less than the number of generalized curvatures, in which case we need to find curves for which some of the generalized curvatures remain constant. Note that this is an issue even in the single-qubit case if only one control field is present. In this circumstance, one needs to restrict to closed curves of constant torsion. How to do this in general for multiple curvatures is an important open question for future work.

\section{ACKNOWLEDGMENTS}

We thank Fei Zhuang for useful discussions. E.B. acknowledges support from the Office of Naval Research (Grant No. N00014-17-1-2971). This work is supported by the Laboratory for Physical Sciences.

[1] A. G. Fowler, M. Mariantoni, J. M. Martinis, and A. N. Cleland, Surface codes: Towards practical large-scale quantum computation, Phys. Rev. A 86, 032324 (2012). 
[2] E. L. Hahn, Spin echoes, Phys. Rev. 80, 580 (1950).

[3] H. Y. Carr and E. M. Purcell, Effects of diffusion on free precession in nuclear magnetic resonance experiments, Phys. Rev. 94, 630 (1954).

[4] S. Alexander, Spin echo method for measuring relaxation times in two-line NMR spectra, Rev. Sci. Instr. 32, 1066 (1961).

[5] L. Viola and S. Lloyd, Dynamical suppression of decoherence in two-state quantum systems, Phys. Rev. A 58, 2733 (1998).

[6] L. Viola, E. Knill, and S. Lloyd, Dynamical Decoupling of Open Quantum Systems, Phys. Rev. Lett. 82, 2417 (1999).

[7] K. R. Brown, A. W. Harrow, and I. L. Chuang, Arbitrarily accurate composite pulse sequences, Phys. Rev. A 70, 052318 (2004).

[8] K. Khodjasteh and D. A. Lidar, Fault-Tolerant Quantum Dynamical Decoupling, Phys. Rev. Lett. 95, 180501 (2005).

[9] W. M. Witzel and S. Das Sarma, Multiple-Pulse Coherence Enhancement of Solid State Spin Qubits, Phys. Rev. Lett. 98, 077601 (2007).

[10] W. M. Witzel and S. Das Sarma, Concatenated dynamical decoupling in a solid-state spin bath, Phys. Rev. B 76, 241303 (2007).

[11] G. S. Uhrig, Keeping a Quantum Bit Alive by Optimized $\pi$-Pulse Sequences, Phys. Rev. Lett. 98, 100504 (2007).

[12] W. Yang and R.-B. Liu, Universality of Uhrig Dynamical Decoupling for Suppressing Qubit Pure Dephasing and Relaxation, Phys. Rev. Lett. 101, 180403 (2008).

[13] B. Lee, W. M. Witzel, and S. Das Sarma, Universal Pulse Sequence to Minimize Spin Dephasing in the Central Spin Decoherence Problem, Phys. Rev. Lett. 100, 160505 (2008).

[14] H. Uys, M. J. Biercuk, and J. J. Bollinger, Optimized Noise Filtration through Dynamical Decoupling, Phys. Rev. Lett. 103, 040501 (2009).

[15] K. Khodjasteh, D. A. Lidar, and L. Viola, Arbitrarily Accurate Dynamical Control in Open Quantum Systems, Phys. Rev. Lett. 104, 090501 (2010).

[16] M. J. Biercuk, A. C. Doherty, and H. Uys, Dynamical decoupling sequence construction as a filter-design problem, Jour. Phys. B. 44, 154002 (2011).

[17] K. Khodjasteh and L. Viola, Dynamically Error-Corrected Gates for Universal Quantum Computation, Phys. Rev. Lett. 102, 080501 (2009).

[18] T. van der Sar, Z. H. Wang, M. S. Blok, H. Bernien, T. H. Taminiau, D. M. Toyli, D. A. Lidar, D. D. Awschalom, R. Hanson, and V. V. Dobrovitski, Decoherence-protected quantum gates for a hybrid solid-state spin register, Nature 484, 82 (2012).

[19] K. Khodjasteh, H. Bluhm, and L. Viola, Automated synthesis of dynamically corrected quantum gates, Phys. Rev. A 86, 042329 (2012).

[20] X. Wang, L. S. Bishop, J. P. Kestner, E. Barnes, K. Sun, and S. Das Sarma, Composite pulses for robust universal control of singlet-triplet qubits, Nat. Commun. 3, 997 (2012).

[21] X. Wang, L. S. Bishop, E. Barnes, J. P. Kestner, and S. Das Sarma, Robust quantum gates for singlet-triplet spin qubits using composite pulses, Phys. Rev. A 89, 022310 (2014).
[22] R. E. Throckmorton, C. Zhang, X.-C. Yang, X. Wang, E. Barnes, and S. Das Sarma, Fast pulse sequences for dynamically corrected gates in singlet-triplet qubits, Phys. Rev. B 96, 195424 (2017).

[23] D. Buterakos, R. E. Throckmorton, and S. Das Sarma, Crosstalk error correction through dynamical decoupling of single-qubit gates in capacitively coupled singlet-triplet semiconductor spin qubits, Phys. Rev. B 97, 045431 (2018).

[24] D. Buterakos, R. E. Throckmorton, and S. Das Sarma, Error correction for gate operations in systems of exchangecoupled singlet-triplet qubits in double quantum dots, Phys. Rev. B 98, 035406 (2018).

[25] S. E. Economou and E. Barnes, Analytical approach to swift nonleaky entangling gates in superconducting qubits, Phys. Rev. B 91, 161405(R) (2015).

[26] X.-H. Deng, E. Barnes, and S. E. Economou, Robustness of error-suppressing entangling gates in cavity-coupled transmon qubits, Phys. Rev. B 96, 035441 (2017).

[27] J. P. Kestner, X. Wang, L. S. Bishop, E. Barnes, and S. Das Sarma, Noise-Resistant Control for a Spin Qubit Array, Phys. Rev. Lett. 110, 140502 (2013).

[28] F. A. Calderon-Vargas and J. P. Kestner, Dynamically Correcting a CNOT Gate for any Systematic Logical Error, Phys. Rev. Lett. 118, 150502 (2017).

[29] J. Zeng, X.-H. Deng, A. Russo, and E. Barnes, General solution to inhomogeneous dephasing and smooth pulse dynamical decoupling, New J. Phys. 20, 033011 (2018).

[30] J. Zeng and E. Barnes, Fastest pulses that implement dynamically corrected single-qubit phase gates, Phys. Rev. A 98, 012301 (2018).

[31] J. Zeng, C. H. Yang, A. S. Dzurak, and E. Barnes, Geometric formalism for constructing arbitrary single-qubit dynamically corrected gates, Phys. Rev. A 99, 052321 (2019).

[32] E. Barnes and S. Das Sarma, Analytically Solvable Driven Time-Dependent Two-Level Quantum Systems, Phys. Rev. Lett. 109, 060401 (2012).

[33] E. Barnes, Analytically solvable two-level quantum systems and landau-zener interferometry, Phys. Rev. A 88, 013818 (2013).

[34] E. Barnes, X. Wang, and S. Das Sarma, Robust quantum control using smooth pulses and topological winding, Sci. Rep. 5, 12685 (2015).

[35] R. E. Throckmorton and S. Das Sarma, Conditions allowing error correction in driven qubits, Phys. Rev. B 99, 045422 (2019).

[36] U. Güngördü and J. P. Kestner, Robust implementation of quantum gates despite always-on exchange coupling in silicon double quantum dots, Phys. Rev. B 101, 155301 (2020).

[37] F. F. Fanchini, J. E. M. Hornos, and R. d. J. Napolitano, Continuously decoupling single-qubit operations from a perturbing thermal bath of scalar bosons, Phys. Rev. A 75, 022329 (2007).

[38] O. E. Dial, M. D. Shulman, S. P. Harvey, H. Bluhm, V. Umansky, and A. Yacoby, Charge Noise Spectroscopy Using Coherent Exchange Oscillations in a Singlet-Triplet Qubit, Phys. Rev. Lett. 110, 146804 (2013).

[39] P. J. J. O'Malley et al., Qubit Metrology of Ultralow Phase Noise Using Randomized Benchmarking, Phys. Rev. Appl. 3, 044009 (2015). 
[40] F. Martins, F. K. Malinowski, P. D. Nissen, E. Barnes, S. Fallahi, G. C. Gardner, M. J. Manfra, C. M. Marcus, and F. Kuemmeth, Noise Suppression Using Symmetric Exchange Gates in Spin Qubits, Phys. Rev. Lett. 116, 116801 (2016).

[41] F. K. Malinowski, F. Martins, Ł. Cywiński, M. S. Rudner, P. D. Nissen, S. Fallahi, G. C. Gardner, M. J. Manfra, C. M. Marcus, and F. Kuemmeth, Spectrum of the Nuclear Environment for GaAs Spin Qubits, Phys. Rev. Lett. 118, 177702 (2017).

[42] M. D. Hutchings, J. B. Hertzberg, Y. Liu, N. T. Bronn, G. A. Keefe, M. Brink, J. M. Chow, and B. L. T. Plourde, Tunable Superconducting Qubits with Flux-Independent Coherence, Phys. Rev. Appl. 8, 044003 (2017).

[43] J. Koch, T. M. Yu, J. Gambetta, A. A. Houck, D. I. Schuster, J. Majer, A. Blais, M. H. Devoret, S. M. Girvin, and R. J. Schoelkopf, Charge-insensitive qubit design derived from the cooper pair box, Phys. Rev. A 76, 042319 (2007).

[44] E. Magesan and J. M. Gambetta, Effective hamiltonian models of the cross-resonance gate, Phys. Rev. A 101, 052308 (2020).

[45] R. Barends, J. Kelly, A. Megrant, D. Sank, E. Jeffrey, Y. Chen, Y. Yin, B. Chiaro, J. Mutus, C. Neill, P. O’Malley, P. Roushan, J. Wenner, T. C. White, A. N. Cleland, and J. M. Martinis, Coherent Josephson Qubit Suitable for Scalable Quantum Integrated Circuits, Phys. Rev. Lett. 111, 080502 (2013).

[46] P. Krantz, M. Kjaergaard, F. Yan, T. P. Orlando, S. Gustavsson, and W. D. Oliver, A quantum engineer's guide to superconducting qubits, App. Phys. Rev. 6, 021318 (2019).

[47] D. C. McKay, S. Sheldon, J. A. Smolin, J. M. Chow, and J. M. Gambetta, Three-Qubit Randomized Benchmarking, Phys. Rev. Lett. 122, 200502 (2019).

[48] P. Mundada, G. Zhang, T. Hazard, and A. Houck, Suppression of qubit crosstalk in a tunable coupling superconducting circuit, Phys. Rev. App. 12, 054023 (2019).

[49] X. Li, T. Cai, H. Yan, Z. Wang, X. Pan, Y. Ma, W. Cai, J. Han, Z. Hua, X. Han, Y. Wu, H. Zhang, H. Wang, Y. Song, L. Duan, and L. Sun, Tunable coupler for realizing a controlled-phase gate with dynamically decoupled regime in a superconducting circuit, Phys. Rev. App. 14, 024070 (2020).

[50] J. Ku, X. Xu, M. Brink, D. C. McKay, J. B. Hertzberg, M. H. Ansari, and B. L. T. Plourde, Suppression of Unwanted ZZ Interactions in a Hybrid Two-Qubit System, Phys. Rev. Lett. 125, 200504 (2020).

[51] M. C. Collodo, J. Herrmann, N. Lacroix, C. K. Andersen, A. Remm, S. Lazar, J.-C. Besse, T. Walter, A. Wallraff, and C. Eichler, Implementation of Conditional Pphase Gates Based on Tunable ZZ Interactions, Phys. Rev. Lett. 125, 240502 (2020).

[52] S. Sheldon, E. Magesan, J. M. Chow, and J. M. Gambetta, Procedure for systematically tuning up cross-talk in the cross-resonance gate, Phys. Rev. A 93, 060302 (2016).

[53] A. D. Patterson, J. Rahamim, T. Tsunoda, P. A. Spring, S. Jebari, K. Ratter, M. Mergenthaler, G. Tancredi, B. Vlastakis, M. Esposito, and P. J. Leek, Calibration of a cross-resonance two-qubit gate between directly coupled transmons, Phys. Rev. App. 12, 064013 (2019).

[54] G. S. Paraoanu, Microwave-induced coupling of superconducting qubits, Phys. Rev. B 74, 140504 (2006).

[55] J. Bylander, S. Gustavsson, F. Yan, F. Yoshihara, K. Harrabi, G. Fitch, D. G. Cory, Y. Nakamura, J.-S. Tsai, and W. D. Oliver, Noise spectroscopy through dynamical decoupling with a superconducting flux qubit, Nat. Phys. 7, 565 (2011).

[56] P. V. Klimov et al., Fluctuations of Energy-Relaxation Times in Superconducting Qubits, Phys. Rev. Lett. 121, 090502 (2018).

[57] J. J. Burnett, A. Bengtsson, M. Scigliuzzo, D. Niepce, M. Kudra, P. Delsing, and J. Bylander, Decoherence benchmarking of superconducting qubits, Npj Quant. Inf. 5, 54 (2019).

[58] M. P. Wardrop and A. C. Doherty, Exchange-based twoqubit gate for singlet-triplet qubits, Phys. Rev. B 90, 045418 (2014).

[59] H. Qiao, Y. P. Kandel, J. S. Van Dyke, S. Fallahi, G. C. Gardner, M. J. Manfra, E. Barnes, and J. M. Nichol, Floquet-Enhanced Spin Swaps, arXiv:2006.10913 (2020).

[60] F. A. Calderon-Vargas, G. S. Barron, X.-H. Deng, A. J. Sigillito, E. Barnes, and S. E. Economou, Fast high-fidelity entangling gates for spin qubits in Si double quantum dots, Phys. Rev. B 100, 035304 (2019).

[61] D. M. Zajac, A. J. Sigillito, M. Russ, F. Borjans, J. M. Taylor, G. Burkard, and J. R. Petta, Resonantly driven CNOT gate for electron spins, Science 359 6374, 439 (2018).

[62] W. Huang, C. H. Yang, K. W. Chan, T. Tanttu, B. Hensen, R. C. C. Leon, M. A. Fogarty, J. C. C. Hwang, F. E. Hudson, K. M. Itoh, A. Morello, A. Laucht, and A. S. Dzurak, Fidelity benchmarks for two-qubit gates in silicon, Nature 569, 532 (2019).

[63] M. Russ, D. M. Zajac, A. J. Sigillito, F. Borjans, J. M. Taylor, J. R. Petta, and G. Burkard, High-fidelity quantum gates in $\mathrm{Si} / \mathrm{SiGe}$ double quantum dots, Phys. Rev. B 97, 085421 (2018).

[64] M. D. Shulman, O. E. Dial, S. P. Harvey, H. Bluhm, V. Umansky, and A. Yacoby, Demonstration of entanglement of electrostatically coupled singlet-triplet qubits, Science 336, 6078 (2012).

[65] X. Wang, E. Barnes, and S. Das Sarma, Improving the gate fidelity of capacitively coupled spin qubits, NPJ Quant. Inf. 1, 15003 (2015).

[66] J. M. Nichol, L. A. Orona, S. P. Harvey, S. Fallahi, G. C. Gardner, M. J. Manfra, and A. Yacoby, High-fidelity entangling gate for double-quantum-dot spin qubits, NPJ Quant. Inf. 3, 3 (2017).

[67] F. A. Calderon-Vargas and J. P. Kestner, Directly accessible entangling gates for capacitively coupled singlet-triplet qubits, Phys. Rev. B 91, 035301 (2015).

[68] H. Bluhm, S. Foletti, D. Mahalu, V. Umansky, and A. Yacoby, Enhancing the Coherence of a Spin Qubit by Operating it as a Feedback Loop That Controls its Nuclear Spin Bath, Phys. Rev. Lett. 105, 216803 (2010).

[69] J. M. Chow, J. M. Gambetta, A. D. Córcoles, S. T. Merkel, J. A. Smolin, C. Rigetti, S. Poletto, G. A. Keefe, M. B. Rothwell, J. R. Rozen, M. B. Ketchen, and M. Steffen, Universal Quantum Gate Set Approaching Fault-Tolerant 
Thresholds with Superconducting Qubits, Phys. Rev. Lett. 109, 060501 (2012).

[70] J. M. Martinis and M. R. Geller, Fast adiabatic qubit gates using only $\sigma_{z}$ control, Phys. Rev. A 90, 022307 (2014).

[71] J. L. Weiner, Closed curves of constant torsion, Archiv der Mathematik 25, 313 (1974).
[72] J. L. Weiner, Closed curves of constant torsion II, Proc. Amer. Math. Soc. 67, 306 (1977).

[73] A. M. Calini and T. A. Ivey, Topology and sine-gordon evolution of constant torsion curves, Phys. Lett. A 254, 170 (1999).

[74] L. M. Bates and O. M. Melko, On curves of constant torsion I, J. Geom. 104, 2 (2013). 